\title{
Two Paired Box 6 mutations identified in Chinese patients with classic congenital aniridia and cataract
}

\author{
YING LIN $^{1 *}$, HONGBIN GAO $^{2,3^{*}}$, YI ZHU ${ }^{1,4^{*}}$, CHUAN CHEN $^{1,4^{*}}$, TAO LI $^{1}$, \\ BINGQIAN LIU ${ }^{1}$, CANCAN LYU $^{1,5}$, YING HUANG ${ }^{1}$, HAICHUN LI $^{1}$, QINGXIU WU $^{1}$, \\ CHENJIN JIN ${ }^{1}$, XIAOLING LIANG ${ }^{1}$, XINHUA HUANG ${ }^{1}$ and LIN LU ${ }^{1}$
}

\author{
${ }^{1}$ State Key Laboratory of Ophthalmology, Zhongshan Ophthalmic Center, Sun Yat-sen University, \\ Guangzhou, Guangdong 510060; ${ }^{2}$ Department of Toxicology, School of Public Health and Tropical Medicine, \\ Southern Medical University, Guangzhou, Guangdong 510515; ${ }^{3}$ Guangdong Laboratory Animals Monitoring Institute, \\ Key Provincial Laboratory of Guangdong Laboratory Animals, Guangzhou, Guangdong 510663, P.R. China; \\ ${ }^{4}$ Department of Molecular and Cellular Pharmacology, University of Miami Miller School of Medicine; ${ }^{5}$ Department of \\ Ophthalmology, Bascom Palmer Eye Institute, University of Miami Miller School of Medicine, Miami, FL 33136, USA
}

Received February 25, 2018; Accepted August 2, 2018

DOI: $10.3892 / \mathrm{mmr} .2018 .9469$

\begin{abstract}
Congenital aniridia is a rare genetic disorder characterized by a variable degree of hypoplasia or absence of iris. It is frequently associated with keratopathy, cataract, juvenile-onset glaucoma and foveal and optic nerve hypoplasia. Mutations in the Paired Box 6 (PAX6) gene on chromosome $11 \mathrm{p} 13$ have been demonstrated to cause aniridia. The aim of the present study was to investigate the genetic variations of PAX6 in two sporadic patients from southern China with classic congenital aniridia and cataract. Complete ophthalmic and physical examinations were performed, including best-corrected visual acuity, intraocular pressure, slit-lamp examination, fundus examination, optical coherence tomography, ultrasound biomicroscopy, and Pentacam scanning. Genomic DNA was extracted from leukocytes of peripheral blood collected from the two patients, their unaffected parents and 200 unrelated control subjects from the same population. Exons 4-13 of the PAX6 gene were amplified by polymerase chain reaction and sequenced directly. Patient 1 was affected with aniridia accompanied by congenital cataract and nystagmus. A novel heterozygous PAX6 frameshift mutation c.277delG (p.Glu93SerfsX31) in exon 6 was identified in
\end{abstract}

Correspondence to: Dr Xinhua Huang or Dr Lin Lu, State Key Laboratory of Ophthalmology, Zhongshan Ophthalmic Center, Sun Yat-Sen University, 54 Xianlie South Road, Guangzhou, Guangdong 510060, P.R. China

E-mail: 1476557150@qq.com

E-mail: drlulin@126.com

*Contributed equally

Key words: aniridia, Paired Box 6 gene, mutation, cataract, glaucoma this patient. Patient 2 was presented with aniridia, congenital cataract, lens subluxation and glaucoma. A recurrent nonsense mutation c.718C $>\mathrm{T}$ (p.Arg240X) in exon 9 was identified in this patient. The present results expand the mutation spectrum of PAX6 and will be valuable for genetic counseling in the affected families. Additionally, the identification of these mutations reiterates the importance of $P A X 6$ in ocular development and sheds light on the pathogenesis of congenital aniridia.

\section{Introduction}

Aniridia is characterized by the congenital hypoplasia or absence of an iris and can be divided into hereditary and sporadic forms (1). Hereditary aniridia is commonly inherited in an autosomal-dominant manner $(1,2)$. Classic aniridia is usually accompanied by a variety of ocular anomalies including keratopathy, lens opacity (cataract), juvenile-onset glaucoma, foveal hypoplasia and optic nerve hypoplasia. Aniridia can also occur as part of the WAGR syndrome (including Wilms tumor, aniridia, genitourinary anomalies and retardation) (3) or Gillespie syndrome (including partial aniridia, ataxia, and intellectual disability) (4). Aniridia occurs in $\sim 1$ per 50,000-100,000 people, with a large range of visual outcomes (5). The visual acuity of the affected individuals largely depends on the extent of the defects in other ocular components. Additionally, children with aniridia frequently present with nystagmus, which leads to decreased light sensitivity (6). Tinted or photochromic lenses can be used to reduce light sensitivity in patients with aniridia, and regular ocular examinations should be performed to closely monitor intraocular pressure and visual acuity (7).

Previous studies have demonstrated that both isolated and syndromic aniridia are commonly associated with mutations in the Paired Box 6 (PAX6) gene on chromosome 11p13 (8-10). The PAX6 gene, highly conserved in both vertebrates and invertebrates, encodes a transcription factor that is a 
master regulator during eye development $(11,12)$. The PAX6 protein contains a paired domain (PD) at the N-terminus, a homeodomain (HD) linked by a glycine-rich domain and a transactivation domain enriched with proline-serine-threonine (PST) residues at the $\mathrm{C}$ terminus. The $\mathrm{PD}$ domain consists of an N-terminal and a $\mathrm{C}$-terminal subdomain, which are important for DNA recognition $(13,14)$. The linker region between the PD and the HD provides a structural base for DNA binding (15). The PST domain, which is rich in proline, serine and threonine, has transactivation function (16). However, the role of PAX6 protein in ocular development is still not fully understood and only a few target genes of PAX6 have been identified (17). So far, $>400$ mutations in the PAX6 gene have been reported. Affected patients with different mutations can present very distinct clinical phenotypes (1). In the present study, two PAX6 mutations were identified from two patients in southern China with classic congenital aniridia and cataract.

\section{Materials and methods}

Study subjects and clinical examination. A total of two sporadic patients were diagnosed as classic congenital aniridia and cataract at Zhongshan Ophthalmic Center (Guangzhou, China). Patient 1 was a 4 year-old male and was recruited in August 2015. Patient 2 was a 24 year-old male and was recruited in March 2015. Visual acuity was examined using the Early Treatment Diabetic Retinopathy Study chart (Precision Vision, La Salle, IL, USA). Intraocular pressure (IOP) was measured by a Goldmann applanation tonometer (Haag-Streit Diagnostics, Koeniz, Switzerland). Anterior segment images were obtained using a BX 900 Slit Lamp (Haag-Streit Diagnostics). Optical coherence tomography (OCT) scans (TOPCON Corporation, Tokyo, Japan) were performed to assess the thickness and morphology of the posterior pole of the retina. Anterior segment dimensions were measured by ultrasound biomicroscopy (UBM; Suoer Electronic Ltd.; Model SW-3200L) and Pentacam ${ }^{\circledR}$ HR version 70700 (OCULUS Optikgeräte GmbH, Wetzlar, Germany). Complete physical examinations were performed to exclude systemic diseases, such as diabetes, hypertension, Wilms tumor and genitourinary anomalies.

Blood sample collection. Venous blood samples from the patients, their unaffected parents, as well as 200 unrelated control subjects from the same population (18-48 years old; male/female $=108 / 92$ ) were collected. Genomic DNA was extracted from peripheral blood leukocytes as described previously $(18,19)$. Briefly, $1 \mathrm{ml}$ of blood sample was collected from each participant, lysed in red blood cell lysis buffer (Sigma Aldrich; Merck KGaA, Darmstadt, Germany) and centrifuged at a speed of 2,000 x $\mathrm{g}$ for $5 \mathrm{~min}$ at room temperature. Genomic DNA was extracted using a DNeasy Blood \& Tissue Kit following the manufacturer's protocol (Qiagen $\mathrm{GmbH}$, Hilden, Germany) $(18,19)$.

Detection of the mutation. Exons of the PAX6 gene (PAX6) were amplified by polymerase chain reaction (PCR) (20). The primer sequences used are listed in Table I. All reagents in the PCR reaction were obtained from a PCR amplification kit
(Takara Bio, Inc., Otsu, Japan). The thermocycling conditions used were as follows: A single $5 \mathrm{~min}$ step at $94^{\circ} \mathrm{C}$; followed by 40 cycles at $94^{\circ} \mathrm{C}$ for $45 \mathrm{sec}, 53-65^{\circ} \mathrm{C}$ for $45 \mathrm{sec}$ and $72^{\circ} \mathrm{C}$ for $45 \mathrm{sec}$; and a final $10 \mathrm{~min}$ step at $72^{\circ} \mathrm{C}$. The PCR products were sequenced from both directions with an ABI3730 Automated Sequencer (Applied Biosystems; Thermo Fisher Scientific, Inc., Waltham, MA, USA). The sequencing results were analyzed using Seqman (version 2.3; Technelysium Pty Ltd., Brisbane, Queensland, Australia) and compared with the reference sequences in the database at the National Center for Biotechnology Information (NCBI; www.ncbi.nlm.nih.gov). The variants identified in the PAX6 gene were summarized using the Leiden Open source Variation Database (LOVD; www.lovd.nl) (21).

Ethics. All experiments were carried out in accordance with the guidelines approved by the ethics committee of Zhongshan Ophthalmic Center at Sun Yat-sen University (Guangzhou, Guangdong, China) and strictly followed the Declaration of Helsinki. Written informed consent was obtained from each participant. All participants provided informed consent for the publication of their clinical and genetic sequencing data in the present study.

\section{Results}

Clinical presentations. The sporadic patients were from the southern part of China. Patient 1 was a 4 -year-old male presented with aniridia, congenital cataract and horizontal nystagmus. He had decreased visual acuity from early childhood. Patient 1 had cataract surgery two years ago and developed posterior capsular opacity characterized by Elschnig pearls in the posterior capsule (Fig. 1). Applanation tonometry demonstrated a normal IOP in both eyes. The corneas were transparent and normal in size, with a width of $10.5 \mathrm{~mm}$ (oculus dexter; OD) and $10.5 \mathrm{~mm}$ (oculus sinister; OS). OCT revealed a normal retina structure (Fig. 2). He did not have other systemic diseases.

Patient 2 was a 24-year-old male with impaired vision and eye pain. The visual acuity (LogMAR) was 0.5 (OD) and 0.7 (OS), which could not be corrected. The width of the cornea was $11.5 \mathrm{~mm}$ (OD) and $11.5 \mathrm{~mm}(\mathrm{OS})$. The intraocular pressure was $22.9 \mathrm{mmHg}(\mathrm{OD})$ and $26.6 \mathrm{mmHg}$ (OS). The axial length of the eyeballs was $26.45 \mathrm{~mm}$ (OD) and $26.51 \mathrm{~mm}(\mathrm{OS})$. Anterior segment imaging exhibited aniridia and cataract. The degree of lens opacity in the left eye was more severe compared with in the right eye. Both eyes exhibited lens subluxation (Fig. 3). UBM demonstrated a shallow anterior chamber with uneven anterior chamber depth (ACD). The central ACD (CACD) was $1.88 \mathrm{~mm}(\mathrm{OD})$ and $0.66 \mathrm{~mm}(\mathrm{OS})$. The anterior chamber angles in both eyes were closed (Fig. 4A-D). Following cataract surgery of the left eye, Pentacam examination demonstrated that the CACD (OS) raised to $4.17 \mathrm{~mm}$ (Fig. 4E and F). The IOP of the left eye decreased to $14.6 \mathrm{mmHg}$ postoperatively.

Mutation screening. In Patient 1, a novel heterozygous mutation c.277delG (p.Glu93SerfsX31) in exon 6 of the PAX6 gene was identified (Fig. 5). This frameshift mutation is predicted to produce a premature stop codon, and thus is likely to produce 

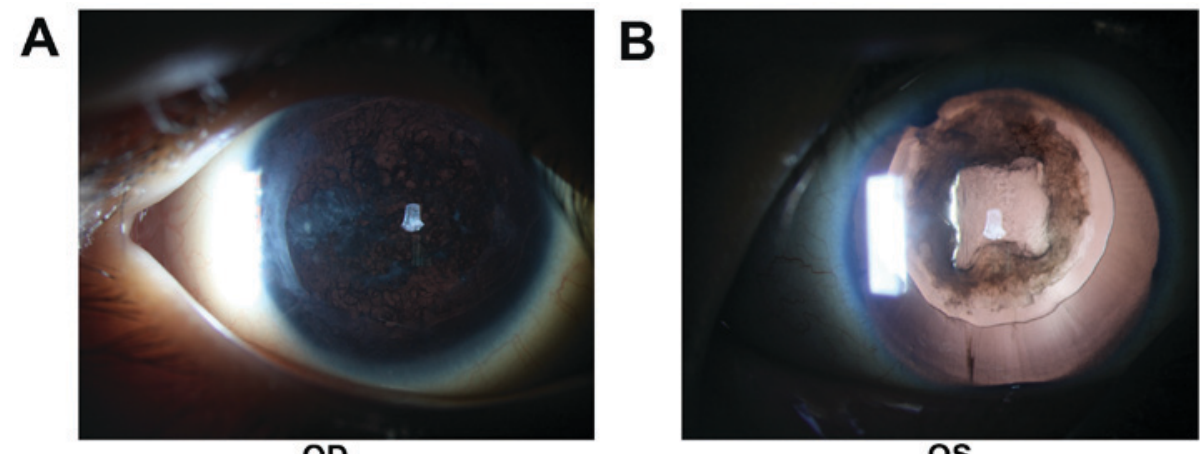

OD

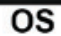

Figure 1. Anterior segment images of Patient 1. Elschnig pearls in the posterior capsule were clearly visible, indicating the development of post-cataract. (A) The right eye and (B), the left eye. OS, oculus sinister; OD, oculus dexter.

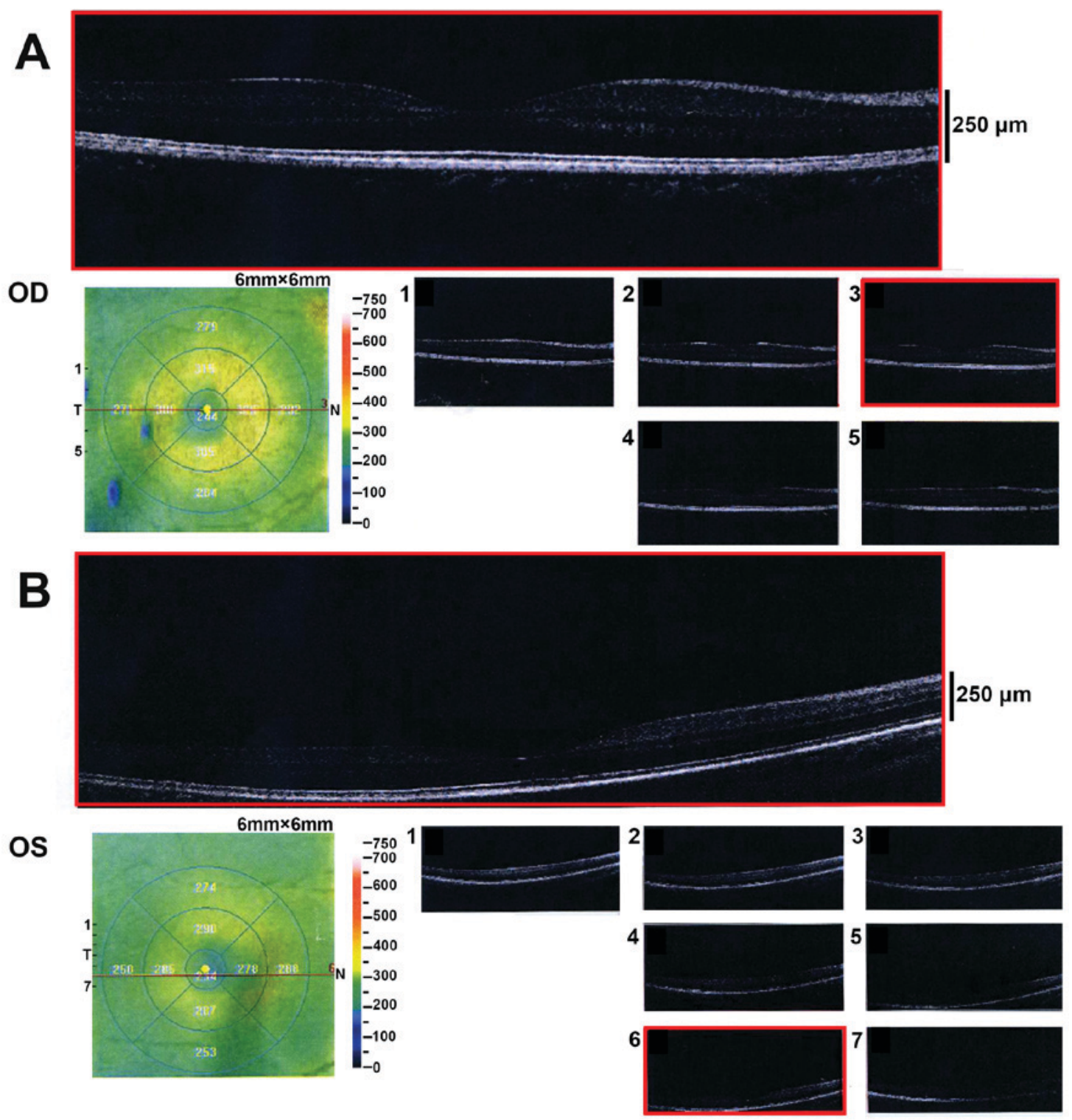

Figure 2. Optical coherence tomography exanimation of Patient 1. The thickness and morphology of the retina were normal. (A) The right eye and (B) the left eye. Numbers 1-7 indicate different representative sections of the optical coherence tomography scans. OD, oculus dexter; OS, oculus sinister.

a truncated protein. In Patient 2, a recurrent nonsense mutation c.718C $>$ T (p.Arg240X) in exon 9 was detected (Fig. 5A). Multiple sequencing alignment indicated that the residues at positions 93 and 240 of the PAX6 protein are highly conserved across species (Fig. 5B). These mutations were not detected in their unaffected parents or the 200 unrelated control subjects 
Table I. Summary of the primers and products size used for the amplification of the PAX6 exons.

\begin{tabular}{lllcc}
\hline Exon & \multicolumn{1}{c}{ Forward (5'-3') } & \multicolumn{1}{c}{ Reverse $\left(5^{\prime}-3^{\prime}\right)$} & $\begin{array}{c}\text { Product } \\
\text { size }(\mathrm{bp})\end{array}$ & Temperature $\left({ }^{\circ} \mathrm{C}\right)$ \\
\hline 4 & TGCAGCTGCCCGAGGATTA & GCACCCCGAGCCCGAAGTC & 144 & 65 \\
5 & TCCCTCTTCTTCCTCTTCACT & GGGGTCCATAATTAGCATC & 301 & 58 \\
$5 \mathrm{a}, 6$ & GCTCTCTACAGTAAGTTCTC & AGGAGAGAGCATTGGGCTTA & 457 & 59 \\
7 & AATCCACCCACTGTCCCG & CCAGCCACCTTCATACCG & 542 & 59 \\
8 & TCAGGTAACTAACATCGCA & GTTGACTGTACTTGGAAGAA & 719 & 53 \\
$9,10,11$ & GAGGTGGGAACCAGTTTGATG & CAAGCCAATCTCTGTAGTGCG & 890 & 55 \\
12 & GCTGTGTGATGTGTTCCTCA & AAGAGAGATCGCCTCTGTG & 245 & 58 \\
13 & CATGTCTGTTTCTCAAAGGG & CCATAGTCACTGACTGAATTAACAC & 202 & 59
\end{tabular}

PAX 6, Paired Box 6.

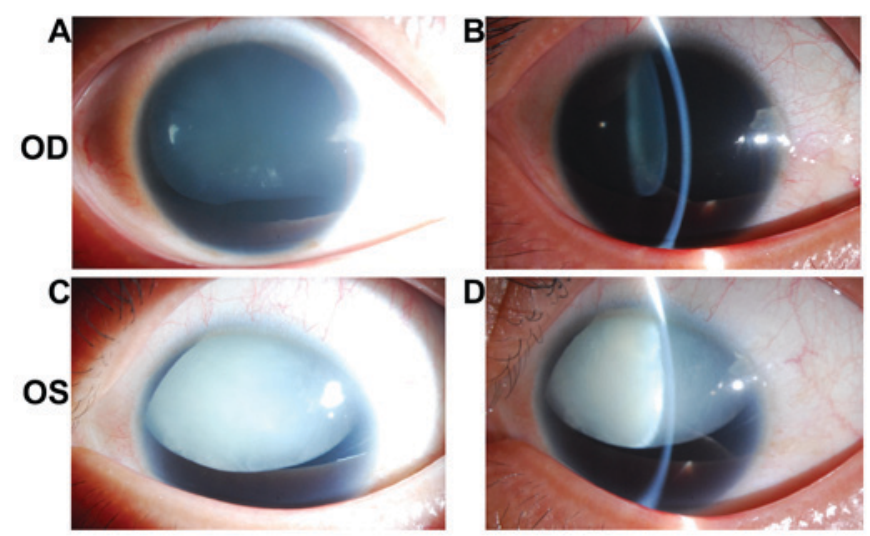

Figure 3. Anterior segment images of Patient 2. The degree of cataract in the left eye was more severe than that in the right eye. Both eyes had lens subluxation. (A) Diffuse illumination of the right eye as revealed by the slit-lamp photo. (B) Slit beam illumination of the right eye as revealed by the slit-lamp photo. (C) Diffuse illumination of the left eye as revealed by the slit-lamp photo. (D) Slit beam illumination of the left eye as revealed by the slit-lamp photo. OD, oculus dexter; OS, oculus sinister.

from the same population. The variants identified in the PAX6 gene are summarized in Fig. 6 (data LOVD; www.lovd.nl).

\section{Discussion}

Mutations in the exons 6 and 9 of PAX6 are commonly identified in patients with congenital aniridia $(22,23)$. In the present study, two mutations in two Chinese patients with classic aniridia and cataract were identified. In Patient 1 , the mutation c.277delG (p.Glu93SerfsX31) in exon 6 is a novel one; two other mutations (c. $277 \mathrm{G}>\mathrm{A}$ and c. $277 \mathrm{G}>\mathrm{T}$ ) have previously been reported at this site $(24,25)$. In Patient 2, the mutation c.718C $>\mathrm{T}$ (p.Arg240X) in exon 9 has been consistently reported according to LOVD. Another mutation c.718delC at this site has been reported recently (26). These two mutations are predicted to result in a premature stop codon and produce a truncated PAX6 protein. The $\mathrm{C}$-terminal region of PAX is enriched in PST residues that are tightly regulated by phosphorylation and serves
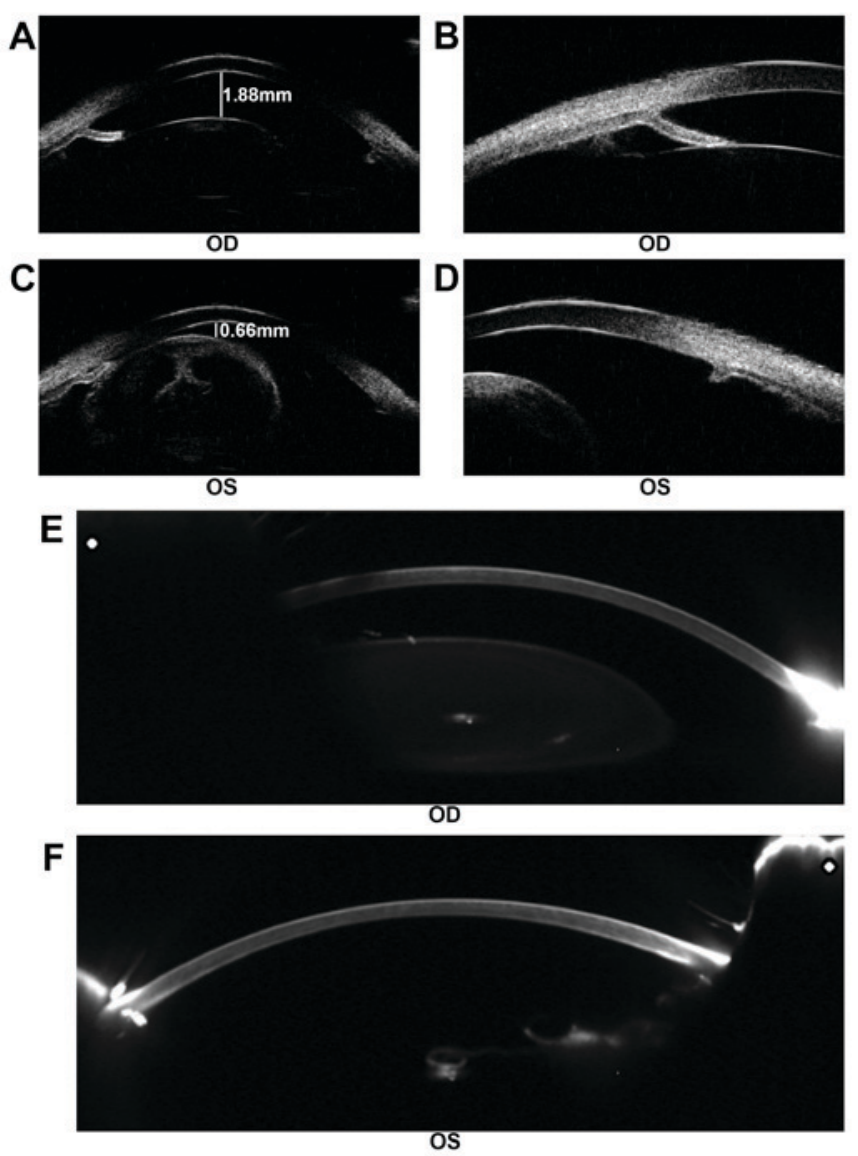

Figure 4. Examination of patient 2 prior to and following surgery. (A) The CACD of the right eye was $1.88 \mathrm{~mm}$, as determined by ultrasound biomicroscopy. (B) The anterior chamber angle of the right eye was closed. (C) The CACD of the left eye prior to cataract surgery was $0.66 \mathrm{~mm}$, as determined by ultrasound biomicroscopy. (D) The anterior chamber angle of the left eye was closed. (E) Pentacam examination of the right eye. (F) Pentacam examination following cataract surgery of the left eye. The CACD (OS) raised to $4.17 \mathrm{~mm}$ postoperatively. $\mathrm{CACD}$, central anterior chamber depth; OD, oculus dexter; OS, oculus sinister.

an essential role in transactivation of downstream targets, such as MAF bZIP transcription factor and GATA binding protein 3 (16). Therefore, the two mutations identified in the 
A
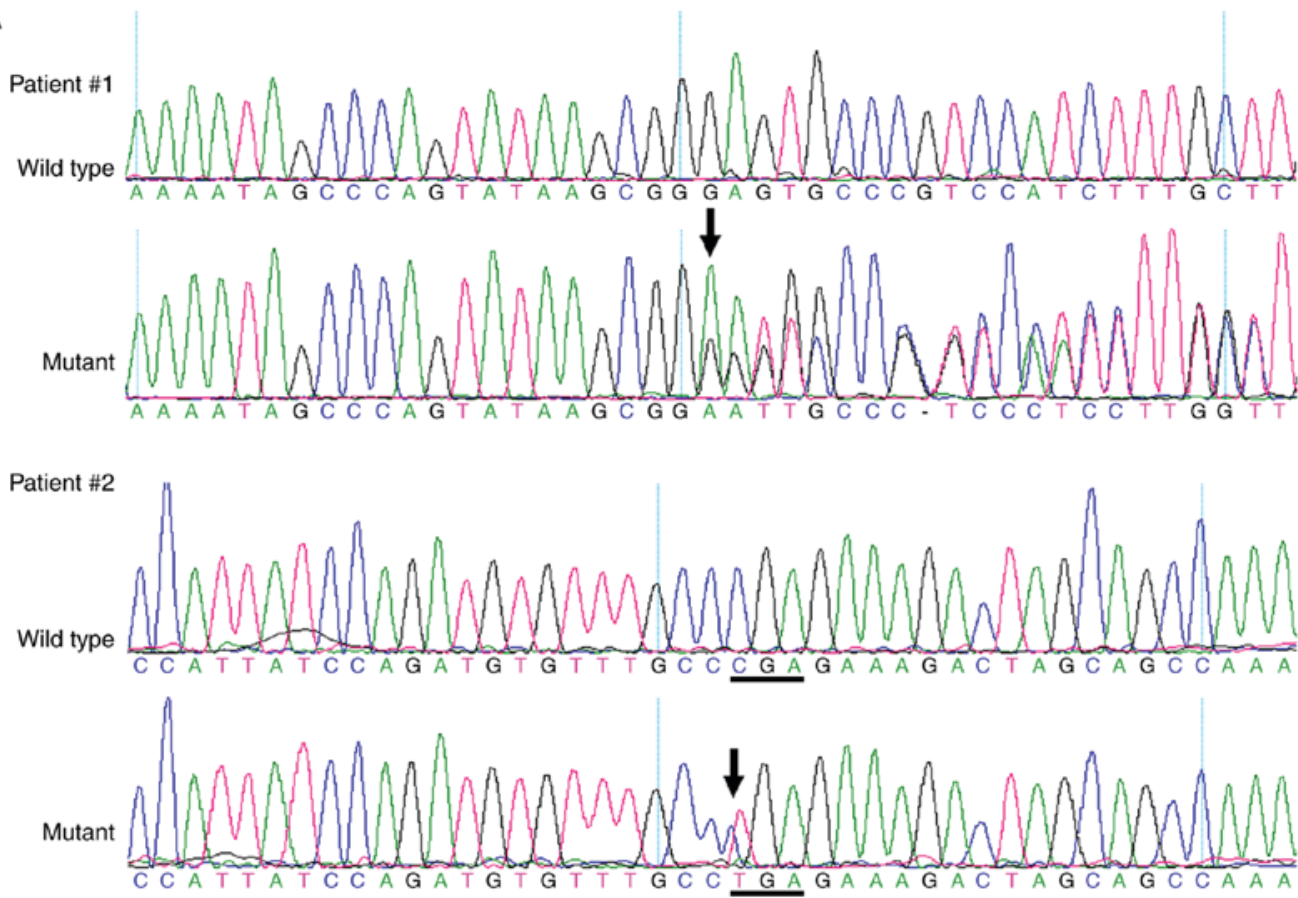

B

\begin{tabular}{|c|c|c|c|c|}
\hline & \multicolumn{2}{|c|}{ Whole sequence identity } & \multirow{2}{*}{$\begin{array}{l}\text { Patient \#1 } \\
93 \\
\nabla\end{array}$} & \multirow{2}{*}{$\begin{array}{l}\text { Patient \#2 } \\
240 \\
\nabla\end{array}$} \\
\hline & DNA & Protein & & \\
\hline H.sapiens & $100 \%$ & $100 \%$ & . . AQYKRECP & PDVFARERLAA . . \\
\hline M.musculus & $94 \%$ & $99 \%$ & . . AQYKRECP & PDVFARERLAA. \\
\hline G.gallus & $88 \%$ & $99 \%$ & . . AQYKRECP & PDVFARERLAA. \\
\hline D.rerio & $82 \%$ & $97 \%$ & . . AQYKRECP & PDVFARERLAA. \\
\hline X.tropicalis & $83 \%$ & $99 \%$ & . . AQYKRECP & PDVFARERLAA. \\
\hline
\end{tabular}

Figure 5. Mutation analysis of the two patients. (A) In Patient 1, a novel heterozygous PAX6 frameshift mutation c.277delG (p.Glu93SerfsX31) in exon 6 was identified. In Patient 2, a recurrent nonsense mutation c.718C >T (p.Arg240X) in exon 9 was identified. (B) Multiple-sequence alignment of the PAX6 genes and PAX6 proteins from different species. The red triangle indicates the location of the mutations. The Glu93 and Arg240 residues are highly conserved across species. PAX6, Paired Box 6.

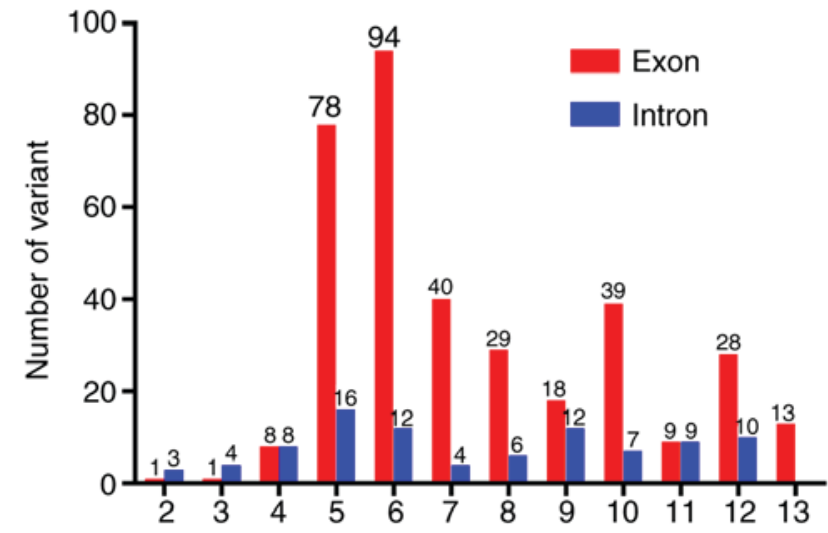

Figure 6. Summary of the number of identified variants in the Paired Box 6 gene. There are also 10 reported cases with a deletion of the whole gene, 6 cases with multiple exon and intron deletions, and 7 cases with variations at the $3^{\prime}$ flanking region.

present study may result in impaired PAX6 function and subsequent abnormal eye development, but this requires further investigation.
Congenital aniridia is commonly accompanied by cataract (in $50-85 \%$ of patients), reflecting an abnormal development of the anterior segment $(27,28)$. Cataracts are rarely presented in infancy, but visually significant lens opacity can develop in the teenage or early adulthood stage $(29,30)$. These patients frequently suffer from severe glare and decreased visual acuity. A number of strategies have been developed to manage the disabling effects of aniridia, including colored contact lens, eyelid surgery, corneal tattooing and implantation of artificial iris (31). For Patient 1, implantation of an artificial iris-lens diaphragm may effectively correct aphakia and reduce glare.

Congenital aniridia is also frequently associated with glaucoma (in $30-50 \%$ of patients) $(27,32)$. Similar to cataract, glaucoma usually develops in late childhood or adulthood, but can also be manifested early in infancy with an enlarged corneal diameter and corneal edema (30). Therefore, IOP needs to be routinely checked in patients with congenital aniridia. Notably, these patients may have increased central corneal thickness, which may lead to a significant overestimation of IOP (33). Therefore, careful and regular examinations of the optic disc morphology by fundoscopy or OCT are required to detect early optic nerve damage. 
In conclusion, two mutations in the PAX6 gene in two sporadic Chinese patients with classic congenital aniridia and cataract were identified. The c.277delG (p.Glu93SerfsX31) mutation in exon 6 is a novel one, while the c.718C $>\mathrm{T}$ (p.Arg240X) mutation in exon 9 is a recurrent one. These findings expand the mutation spectrum of PAX6 and may be valuable for future genetic counseling, and prenatal diagnosis in families with aniridia, but larger studies are needed in order to confirm the above findings.

\section{Acknowledgements}

Not applicable.

\section{Funding}

The present study was supported by the National Natural Science Foundation of China (grant nos. 81500709, 81570862 and 81670872), Guangzhou Science and Technology Project (grant no. 2014Y2-00064), and the State Scholarship Fund from the China Scholarship Council.

\section{Availability of data and materials}

The analyzed datasets generated during the study are available from the corresponding author upon reasonable request.

\section{Authors' contributions}

$\mathrm{XH}, \mathrm{YL}, \mathrm{CC}, \mathrm{YZ}, \mathrm{TL}, \mathrm{CJ}, \mathrm{XL}$ and LL analyzed and interpreted the patient data. HG, BL, CL, YH, QW and HL examined the patients and performed polymerase chain reaction and gene sequence analysis. YL, CC and YZ interpreted the sequencing data. All authors read and approved the final manuscript.

\section{Ethics approval and consent to participate}

All experiments were carried out in accordance with the guidelines approved by the ethics committee of Zhongshan Ophthalmic Center at Sun Yat-sen University (Guangzhou, Guangdong, China) and strictly followed the Declaration of Helsinki. Written informed consent was obtained from each subject.

\section{Patient consent for publication}

All participants provided informed consent for the publication of their clinical and genetic sequencing data in the present study.

\section{Competing interests}

The authors declare that they have no competing interests.

\section{References}

1. Hanson IM, Seawright A, Hardman K, Hodgson S, Zaletayev D, Fekete $\mathrm{G}$ and van Heyningen V: PAX6 mutations in aniridia. Hum Mol Genet 2: 915-920, 1993.

2. Chen JH, Lin W, Sun G, Huang C, Huang Y, Chen H, Pang CP and Zhang M: A novel PAX6 deletion in a Chinese family with congenital aniridia. Mol Vis 18: 989-995, 2012.
3. Fischbach BV, Trout KL, Lewis J, Luis CA and Sika M: WAGR syndrome: A clinical review of 54 cases. Pediatrics 116: 984-988, 2005.

4. Sarsfield JK: The syndrome of congenital cerebellar ataxia, aniridia and mental retardation. Dev Med Child Neurol 13: 508-511, 1971.

5. Hingorani $M$ and Moore A: Aniridia. In: GeneReviews [Internet]. Adam MP, Ardinger HH, Pagon RA, Wallace SE, Bean LJH, Stephens K and Amemiya A (eds). University of Washington, Seattle, WA, 1993-2018.

6. Calvão-Pires P, Santos-Silva R, Falcão-Reis F and Rocha-Sousa A: Congenital aniridia: Clinic, genetics, therapeutics, and prognosis. Int Sch Res Notices 2014: 305350, 2014

7. Lee H, Khan R and O'Keefe M: Aniridia: Current pathology and management. Acta Ophthalmol 86: 708-715, 2008.

8. Yokoi T, Nishina S, Fukami M, Ogata T, Hosono K, Hotta Y and Azuma N: Genotype-phenotype correlation of PAX6 gene mutations in aniridia. Hum Genome Var 3: 15052, 2016.

9. Jordan T, Hanson I,Zaletayev D, Hodgson S, Prosser J, Seawright A, Hastie $\mathrm{N}$ and van Heyningen V: The human PAX6 gene is mutated in two patients with aniridia. Nat Genet 1: 328-332, 1992.

10. Axton R, Hanson I, Danes S, Sellar G, van Heyningen V and Prosser J: The incidence of PAX6 mutation in patients with simple aniridia: An evaluation of mutation detection in 12 cases. J Med Genet 34: 279-286, 1997.

11. Ton CC, Hirvonen H, Miwa H, Weil MM, Monaghan P, Jordan T, van Heyningen V, Hastie ND, Meijers-Heijboer H, Drechsler M, et al: Positional cloning and characterization of a paired box- and homeobox-containing gene from the aniridia region. Cell 67: 1059-1074, 1991.

12. Halder G, Callaerts P and Gehring WJ: Induction of ectopic eyes by targeted expression of the eyeless gene in Drosophila. Science 267: 1788-1792, 1995.

13. Czerny T, Schaffner G and Busslinger M: DNA sequence recognition by Pax proteins: Bipartite structure of the paired domain and its binding site. Genes Dev 7: 2048-2061, 1993.

14. Mishra R, Gorlov IP, Chao LY, Singh S and Saunders GF: PAX6, paired domain influences sequence recognition by the homeodomain. J Biol Chem 277: 49488-49494, 2002.

15. Xu HE, Rould MA, Xu W, Epstein JA, Maas RL and Pabo CO: Crystal structure of the human Pax6 paired domain-DNA complex reveals specific roles for the linker region and carboxy-terminal subdomain in DNA binding. Genes Dev 13: 1263-1275, 1999.

16. Singh S, Chao LY, Mishra R, Davies J and Saunders GF: Missense mutation at the C-terminus of PAX6 negatively modulates homeodomain function. Hum Mol Genet 10: 911-918, 2001.

17. Coutinho P, Pavlou S, Bhatia S, Chalmers KJ, Kleinjan DA and van Heyningen V: Discovery and assessment of conserved Pax6 target genes and enhancers. Genome Res 21: 1349-1359, 2011.

18. Lin Y, Li T, Ma C, Gao H, Chen C, Zhu Y, Liu B, Lian Y, Huang Y, $\mathrm{Li} \mathrm{H}$, et al: Genetic variations in Bestrophin-1 and associated clinical findings in two Chinese patients with juvenile-onset and adult-onset best vitelliform macular dystrophy. Mol Med Rep 17: 225-233, 2018

19. Huang X, Lin Y, Chen C, Zhu Y, Gao H, Li T, Liu B, Lyu C, Huang $\mathrm{Y}, \mathrm{Wu} \mathrm{Q}$, et al: Targeted next-generation sequencing identifies two novel COL2A1 gene mutations in Stickler syndrome with bilateral retinal detachment. Int J Mol Med 42: 1819-1826, 2018.

20. Lin Y, Liu X, Yu S, Luo L, Liang X, Wang Z, Chen C, Zhu Y, Ye S, Yan H and Liu Y: PAX6 analysis of two sporadic patients from southern China with classic aniridia. Mol Vis 18: 2190-2194, 2012.

21. Fokkema IF, Taschner PE, Schaafsma GC, Celli J, Laros JF and bden Dunnen JT: LOVD v. 2.0: the next generation in gene variant databases. Hum Mutat 32: 557-563, 2011.

22. Azuma N, Hotta Y, Tanaka H and Yamada M: Missense mutations in the PAX6 gene in aniridia. Invest Ophthalmol Vis Sci 39: 2524-2528, 1998.

23. Dharmaraj N, Reddy A, Kiran V, Mandal A, Panicker S and Chakrabarti S: PAX6 gene mutations and genotype-phenotype correlations in sporadic cases of aniridia from India. Ophthalmic Genet 24: 161-165, 2003.

24. Villarroel CE, Villanueva-Mendoza C, Orozco L, Alcántara-Ortigoza, MA, Jiménez DF, Ordaz JC and González-del Angel A: Molecular analysis of the PAX6 gene in Mexican patients with congenital aniridia: Report of four novel mutations. Mol Vis 14: 1650-1658, 2008.

25. Park SH, Kim MS, Chae H, Kim Y and Kim M: Molecular analysis of the PAX6 gene for congenital aniridia in the Korean population: Identification of four novel mutations. Mol Vis 18: 488-494, 2012 
26. Pérez-Solórzano S, Chacón-Camacho OF, Astiazarán MC, Ledesma-Gil G and Zenteno JC: PAX6 allelic heterogeneity in Mexican congenital aniridia patients: Expanding the mutational spectrum with Seven novel pathogenic variants. Clin Exp Ophthalmol 45: 875-883, 2017.

27. Angmo D, Jha B and Panda A: Congenital aniridia. J Curr Glaucoma Pract 5: 01-13, 2011.

28. Neuhann IM and Neuhann TF: Cataract surgery and aniridia. Curr Opin Ophthalmol 21: 60-64, 2010.

29. Hashmani S, Haider SI, Khatri B and Rind GK: Management of Aniridia with Ectopia Lentis. Pak J Ophthalmol 31: 48-50, 2015.

30. Parekh M, Poli B, Ferrari S, Teofili C and Ponzin D (eds): Aniridia. Springer, New York, NY, 2015.
31. Adeoti CO, Afolabi AA, Ashaye AO and Adeoye AO: Bilateral sporadic aniridia: Review of management. Clin Ophthalmol 4: 1085-1089, 2010.

32. Brémond-Gignac D: Glaucoma in aniridia. J Fr Ophtalmol 30: 196-199, 2007 (In French).

33. Park SH, Park YG, Lee MY and Kim MS: Clinical features of Korean patients with congenital aniridia. Korean J Ophthalmol 24: 291-296, 2010.

(c) () $\odot$ This work is licensed under a Creative Commons

Attribution-NonCommercial-NoDerivatives 4.0 International (CC BY-NC-ND 4.0) License. 